\title{
NavigaTorch: Projection-based Robot Control Interface using High-speed Handheld Projector
}

\author{
Takefumi Hiraki \\ Osaka University \\ hiraki@sens.sys.es.osaka- \\ u.ac.jp
}

\author{
Shogo Fukushima \\ The University of Tokyo \\ shogo@nae-lab.org
}

\author{
Yoshihiro Kawahara \\ The University of Tokyo \\ kawahara@akg.t.u- \\ tokyo.ac.jp
}

\author{
Takeshi Naemura \\ The University of Tokyo \\ naemura@nae-lab.org
}

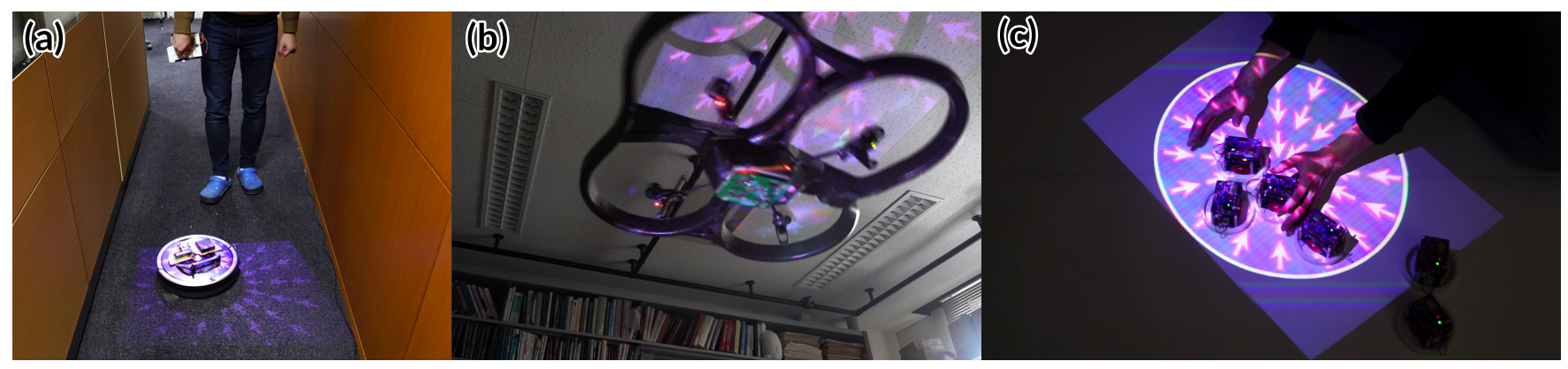

Figure 1: (a) Sweep by Roomba: Application for controlling a household cleaning robot. (b) Drone Navigation: Application for manipulating position and height of a drone. (c) Gathering Swarm: Application for controlling robot swarms.

\begin{abstract}
We propose "NavigaTorch", a projection-based robot control interface that enables the user to operate a robot quickly and intuitively. The user can control and navigate a robot from the third-person viewpoint using the projected video as visual feedback. We achieved flickerless image feedback and a quick response in robot operation by developing a handheld pixel-level visible light communication (PVLC) projector. Our contribution is the development of a robot control interface based on high-speed projection technology and the exploration of the design methodology of projection-based robot control using a handheld projector.
\end{abstract}

\section{CCS CONCEPTS}

- Human-centered computing $\rightarrow$ Interaction devices.

\section{KEYWORDS}

robot control interface, projection-based control, handheld projector, high-speed projection, visible light communication

\section{ACM Reference Format:}

Takefumi Hiraki, Shogo Fukushima, Yoshihiro Kawahara, and Takeshi Naemura. 2019. NavigaTorch: Projection-based Robot Control Interface using High-speed Handheld Projector. In SA '19 Emerging Technologies, November

Permission to make digital or hard copies of all or part of this work for personal or classroom use is granted without fee provided that copies are not made or distributed for profit or commercial advantage and that copies bear this notice and the full citation on the first page. Copyrights for components of this work owned by others than ACM must be honored. Abstracting with credit is permitted. To copy otherwise, or republish, to post on servers or to redistribute to lists, requires prior specific permission and/or a fee. Request permissions from permissions@acm.org.

SA '19 Emerging Technologies, November 17-20, 2019, Brisbane, QLD, Australia

(c) 2019 Association for Computing Machinery.

ACM ISBN 978-1-4503-6942-8/19/11 ..\$15.00

https://doi.org/10.1145/3355049.3360538
17-20, 2019, Brisbane, QLD, Australia. ACM, New York, NY, USA, 3 pages. https://doi.org/10.1145/3355049.3360538

\section{INTRODUCTION}

Robots are becoming increasingly common in everyday life, and many people want to be able to operate these robots in a simple and intuitive matter. Controllers that use the joystick metaphor to specify speed and movement direction are common; these are interfaces using the first-person viewpoint for operation. However, this is counterintuitive because the observation viewpoint and the operation viewpoint are different when the user observes the robot from the third-person viewpoint. In addition, when the user desires to operate many robots in the environment, the operation of selecting the target robot becomes abstract and cumbersome. Projection-based control using a handheld projector is promising because both the observation viewpoint and the operation viewpoint are third-person perspectives, and the position and control target can both be directly specified and operated.

This type of robot control interface has usually been realized by a projector-camera system [Hosoi et al. 2007], but the alignment and calibration of these devices are cumbersome. PICOntorl [Schmidt et al. 2012] tackled this problem by controlling the device by communication using the light projected by a handheld projector. However, flicker was observed in the projected images, and the operation response was poor because a conventional low refresh-rate projector was used.

In this paper, we propose "NavigaTorch", which is a projectionbased robot control interface using a handheld pixel-level visible light communication (PVLC) projector. PVLC superimposes data patterns on pixels with human-imperceptible flicker using a highspeed digital light processing (DLP) projector [Kimura et al. 2008]. PVLC can display a visual image containing superimposed data 

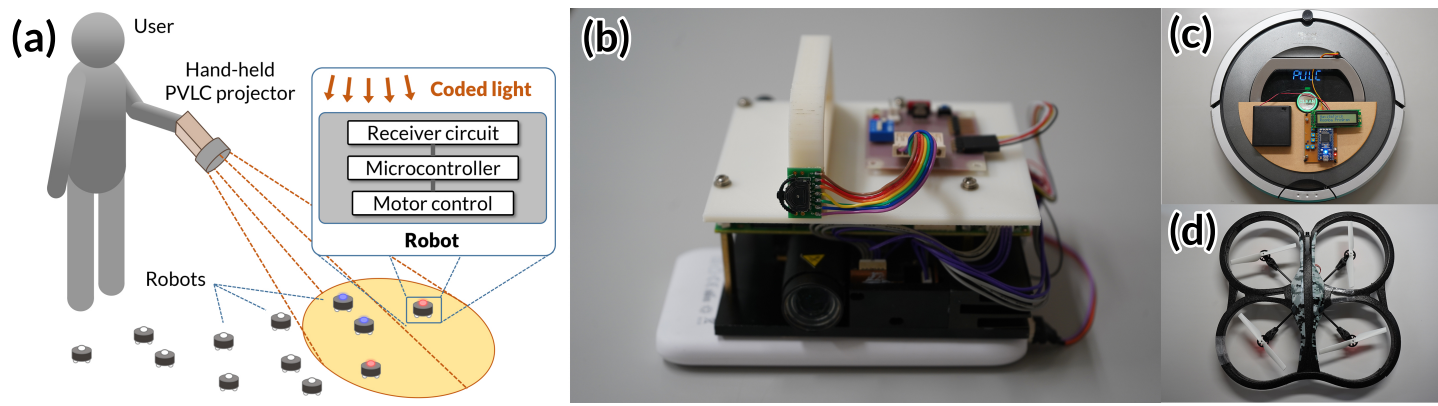

(e)

Figure 2: (a) System configuration of NavigaTorch. (b) Handheld PVLC projector system. (c) Instrumented household robot. (d) Instrumented drone. (e) Instrumented small robot for swarm control.

as bit patterns that are decodable by receiver circuits. We can realize flickerless image projection and robot operation with quick response thanks to the rapid update of video and information using this high-speed handheld projector. The user can implicitly and intuitively select target robots to control by directly projecting images onto those robots. In addition, the user can navigate the robots in a smooth and intuitive manner by moving the projector itself while viewing the projected images as visual feedback.

\section{SYSTEM DESIGN}

The proposed system, shown in Figure 2a, comprises a handheld projector that projects video and information and a robot that receives this information. We developed a handheld system using the high-speed DLP projector (DLP LightCrafter 4500, Texas Instruments) (Figure 2b), which is compact, lightweight (approximately $500 \mathrm{~g}$ ), and can be battery-driven. We generated an array containing the data to be embedded in binary form, representing each bit as a pixel. We combined this array with a visual image into a 24-bit bitmap file and transferred it to the memory of the projector. The microcomputer communicates with the projector and realizes PVLC by projecting the binary images of the data and the visual image frames at appropriate times. The microcomputer also monitors the input of the lever switch of the handle and switches the projection images and embedded information.

We developed three types of robots: household robots, drones, and small robots for swarm control (Figure 2c-e). Each has a lightreceiving circuit capable of receiving high-speed blinking lights. As this circuit filters only the signal light, the robot can operate in an everyday lighting environment. We developed the household robot based on Roomba 760 (iRobot), AR.Drone 2.0 (Parrot), and the small robots based on our previous work [Hiraki et al. 2018].

We explored the design methodology of projection-based robot control using a handheld projector. This methodology comprises the projection image, the control information to embed, and the actions of the robots that receive it. The system embeds the velocity vector field as control information in the images and projects the video that represents this field visually. The user can control the behavior of the robots while obtaining visual feedback. We selected three velocity vector fields (one way, convergence, and divergence) regarding the artificial potential field method [Arkin 1989]; the system realizes various robot operations by switching these fields. Position coordinate information, to estimate the orientation of robots, and function information, to control the function of robots, are also embedded in the projected images.

\section{APPLICATIONS}

We demonstrated three robot control applications using the household robot, drone, and robot swarms. Sweep by Roomba is an application that allows the user to intuitively operate the household cleaning robot (Figure 1a). By projecting the image onto the robot, the user can instruct the robot to clean a specific place and guide its return to the base location. Drone Navigation application allows the user to control the position and height of a drone (Figure 1b). The user can control the position of the drone by moving the image, and the height of the drone can be controlled using the lever switch. Gathering Swarm application enables users to operate small robots as swarms (Figure 1c). The user can select the operation target by projecting the image only to the robot that the user wants to operate. To select the robot to operate, we can adjust the projected area of the image by changing the distance between the interface and the robot or by switching the image.

\section{CONCLUSION}

We propose "NavigaTorch", which is a flickerless and responsive projection-based robot control interface. In future work, we intend to build a standalone system that can generate projection images dynamically with a single-board computer.

\section{ACKNOWLEDGMENTS}

This work was supported by JST ERATO Grant Number JPMJER1501, JSPS KAKENHI Grant Numbers JP17J04216, JP19J00101, Japan.

\section{REFERENCES}

Ronald C. Arkin. 1989. Motor Schema - Based Mobile Robot Navigation. The International fournal of Robotics Research 8, 4 (aug 1989), 92-112.

Takefumi Hiraki, Shogo Fukushima, Yoshihiro Kawahara, and Takeshi Naemura. 2018. Phygital Field: An Integrated Field with Physical Robots and Digital Images Using Projection-Based Localization and Control Method. Fournal of Control, Measurement, and System Integration 11, 4 (2018), 302-311.

Kazuhiro Hosoi, Vinh Ninh Dao, Akihiro Mori, and Masanori Sugimoto. 2007. VisiCon: A Robot Control Interface for Visualizing Manipulation Using a Handheld Projector. In Proceedings of 2007 International Conference on Advances in computer entertainment technology - ACE '07. 99-106.

Sho Kimura, Ryo Oguchi, Hideo Tanida, Yasuaki Kakehi, Keita Takahashi, and Takeshi Naemura. 2008. PVLC Projector: Image Projection with Imperceptible Pixel-Level Metadata. In ACM SIGGRAPH 2008 Posters on - SIGGRAPH '08. Article No.135. 
Dominik Schmidt, David Molyneaux, and Xiang Cao. 2012. PICOntrol: Using a Handheld Projector for Direct Control of Physical Devices through Visible Light. In
Proceedings of the 25th annual ACM symposium on User interface software and technology - UIST '12. 379-388. 